\title{
Vehicle Monitoring and Routing System
}

\author{
Mit K. Shah \\ K.J. Somaiya College of \\ Engineering \\ Marine Lines, Mumbai-02 \\ Maharashtra India
}

\author{
Parshva N. Shah \\ K.J. Somaiya College of \\ Engineering \\ Ghatkopar, Mumbai-86 \\ Maharashtra India
Smita R. Sankhe
Assistant Professor
Engineering \\ K.J. Somaiya College of
}

\author{
Khyati M. Thakkar \\ K.J. Somaiya College of \\ Engineering \\ Ghatkopar, Mumbai-75 \\ Maharashtra India
}

\begin{abstract}
In this paper, a vehicle monitoring and routing system has been proposed which allows a vehicle administrator to monitor the vehicle in real-time using a GPS-based device possessed by its driver. This system can continuously track a vehicle and show its exact position using GPS. The proposed system is also capable of finding the shortest route to reach the destination which uses the proposed algorithm to find the same. This system also possesses the facility of speed monitoring and giving alerts to the driver if he over speeds his vehicle. Also, the system gives accident alerts to the vehicle admin.
\end{abstract}

\section{General Terms}

Vehicle monitoring, Routing system, GPS tracking.

\section{Keywords}

Real-time monitoring, routing, speed monitoring and alerts, accident alerts.

\section{INTRODUCTION}

In today's world GPS (Global positioning system) is the most widely used technology in vehicle monitoring and routing system. Security of public and private vehicles like school buses is of prime importance. There are various cases encountered in recent times where the children don't reach home in time or the vehicles loaded with goods are hijacked. Because of which the parents or the vehicle owners get worried. GPS technology can be used to solve this problem. Using GPS and GIS (Global information system) we can find the exact location of the vehicle.

Time is an important factor considered while travelling. Reaching on destination as soon as possible is always admirable. Google Maps provides all the routes from a source to destination. The routes provided by Google Maps may not be the shortest/fastest route available. There needs to be a system devised which can help a person to reach from source to destination in minimal amount of time. An efficient algorithm is devised for these purposes.

In recent times, the rate of road accidents has increased considerably. For helping the victims of road accidents, a quick service should be provided. An alert system module is incorporated for this purpose. This module is based on GPS, GPRS/GSM technology. A GSM mobile device with active GPRS connection is installed in the vehicle. The position of the vehicle which is received through GPS is sent to the concerned person and to the ambulance hotline number through SMS (Short Message Service). Immediate attention will be provided to the victim through the proposed system.

\section{LITERATURE SURVEY}

S. Sivakumar and Dr. C.Chandrasekar formulated a real-time algorithm called Modified Dijkstra's algorithm whose basic idea was to modify the Dijkstra's algorithm to make it suitably useful for finding shortest route between source and destination considering other real-time factors like traffic congestion, time and distance. They developed a tool using Java and compared the proposed Modified Dijkstra's algorithm with the existing algorithms like DKA( (Dijkstra's algorithm with Approximate Buckets) on Jaipur database. Comparisons were made based on number of nodes visited and time taken to reach the solution [1].

Another author Liang Dai made a comparison between Dijkstra's algorithm and A* algorithm by implementing both of them on Ottawa city road network. He compared the running times of both the algorithms and concluded that $\mathrm{A}^{*}$ can have better running time than Dijkstra's if it uses Eucledian distance as its heuristic function. Though, their time complexities are almost same, they may be chosen depending on the road network chosen [2].

A.Renugambal and V.Adilakshmi Kameswari made an android application which can be run on GPS-driven mobile phones equipped by the taxi drivers. These phones send their location via GPS which also has the capacity of finding an optimal route by using min-max algorithm [3].

Paul Benjamin Et Al designed a GPS-based vehicle monitoring and alert system. They included a management system, fuel usage monitor and an onboard location display along with an accident and robbery alert system. Their project used Google Maps API, SMS gateway server which triggers sending SMS to nearby hospital when the inbuilt air-bags open, a panic button that can be pressed by the driver which is easily accessible to him that sends an SMS to a nearby police station. They formulated a C\# code for sending location through GPS to the database [4].

M. A. Hannanet Et Al implemented a bus monitoring system which used radio frequency identification tag (RFID) along with GPS, GPRS and GIS to monitor bus. They used an RFID reader which continuously sends an operating energy or isotropic radiated power to the RFID tag. Based on the calculated distance between the tag and the reader, it is decided whether to obtain the data from the tag or not and whether to send data to the control center or not. The time of the arrivals of the readings can be recorded too along with 
other processed data which is saved in the database. Then, this data is shown to the bus drivers [5].

Fleischer Et Al developed a GPS/GSM based vehicle alert and tracking system for inter-city buses and goods transportation. They provided real time tracking and alert mechanisms for accident and robbery. Google maps APIs where used to show maps. Airbag sensing mechanism would help to provide information related to accident. In case of robbery, they gave alert button, that when pressed will send SMS to nearby police station. All these was done with the help of C\# code [6].

\section{PROBLEM DEFINITION}

Daily, drivers face problems while travelling on highways, unknown routes and different terrains. Some more problems might be of spare settlement, carjacking or accidents. When the vehicle leaves from its source for its journey, until he reaches its destination, an analyst may rely completely on the previous reports or $\log$ that were generated. No real time information regarding the state of vehicle can ever be transmitted to the analyst. So real time tracking of the vehicle is necessary for safety and security purpose.

Real time monitoring of the vehicle is important these days due to an increasing number of accidents and robbery. Whenever such an incident takes place, the vehicle admin if monitoring on real time basis, can immediately notify nearby hospitals or police stations according to the situation he will be notified with. Also, the parents of school going kids can be informed so that they can take a sigh or for the owner of the good being travelled via transport vehicle.
VMARS is a project that covers most important and rare feature of alerts. Succinctly put, the developed system will be able to (1) Provide real-time tracking and monitoring of the vehicle. (2) Show the shortest path to the driver by applying the proposed algorithm considering all the real-time factors. (3) Alerting the driver whenever he exceeds the safe speed. (4) Proving quick and timely alert to the vehicle admin to take actions in case of an accident.

\section{PROPOSED SYSTEM DESIGN AND METHODOLOGY}

The proposed system consists of GPS module, GSM modem, SMS Server and GPRS Server. The System continuously tracks the vehicle through a GSM device with GPS enabled in the device. The management system receives information from the vehicle via a SMS gateway server which is installed at the server side i.e. vehicle monitoring admin. The SMS is sent through VPN (Virtual Private Network). SMS is used to inform clients about any delay or other important notifications. Driver sends an SMS to vehicle monitoring admin requesting for alternate route in case of a rally or unexpected blockage of a road or if any accident has occurred blocking the road.

The System architecture and how the various components of the system interact with each other are shown in the diagram below:

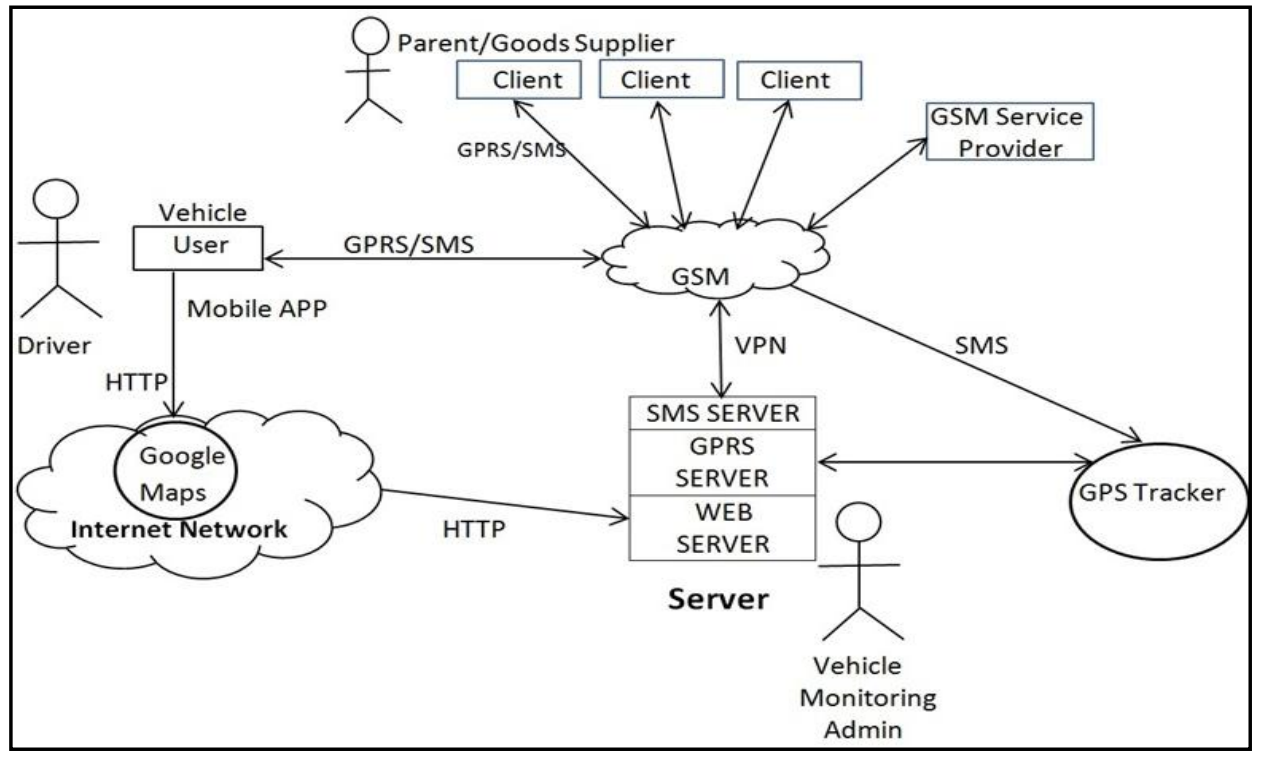

Fig 1: Proposed system design and architecture.

\section{PROPOSED ALGORITHM}

The vehicle routing problem is a different from normal shortest path problem, which are having links that will represent road maps with its junctions.

If a graph is considered as an analogy to the road network where junctions are nodes and roads are edges, and if only distance is considered as a factor for finding the shortest path between two nodes, normal Dijkstra's algorithm is proven to be the best algorithm for this purpose. But when real-time road network is considered, some other factors like road congestion, time of travel, etc. have to be considered too. For this purpose, a modified Dijkstra's algorithm can be formulated which considers other factors too which are mandatory for finding shortest path in real-time road network.

The available time to provide an alternate path must be limited due to road network constraints. It should take very less time to provide an alternate path.

\subsection{Modified Dijkstra's Shortest Path Algorithm (MDSP)}

This algorithm shows better results than the existing Dijkstra's shortest path algorithm on real time road network. 
This algorithm considers multiple parameters like time, distance and congestion for finding possible shortest route from the source to destination. The algorithm's pseudo code is as below:

// Let source be the origin vertex and initialize Visited and ShortestDistance $[u]$ as

Visited $:=\{$ source $\}$

ShortestDistance[source] $:=0$

// Let user choose any preference among Distance, Time and Congestion factors

\section{ACCEPT Choice}

// Update distance between every nodes as per their corresponding factors

FOR each vertex pair $[\mathrm{u}, \mathrm{v}]$

Case Distance: //Do nothing

Case Time: //Update according to time factor Distance $[\mathrm{u}, \mathrm{v}]:=$ Distance $[\mathrm{u}, \mathrm{v}] *$ time

factor

Case Congestion: //Update according to congestion factor congestion factor

$$
\text { Distance }[\mathrm{u}, \mathrm{v}]:=\text { Distance }[\mathrm{u}, \mathrm{v}] \text { * }
$$

\section{END FOR}

FOR each vertex in $\mathrm{V}-\{$ source $\}$

$$
\text { ShortestDistance[u] := Distance[source, } \mathrm{u}]
$$

\section{END FOR}

//Add vertices to Visited until Visited includes all vertices in $V$ WHILE Visited not equal to $\mathrm{V}$

// Find the vertex $w$ among remaining vertices closest to the source

MinimumDistance $:=$ INFINITE
FOR each $v$ in remaining vertices

IF ShortestDistance[v] <

MinimumDistance

MinimumDistance $=$

ShortestDistance[v]

$$
\mathrm{w}:=\mathrm{v}
$$

\section{END FOR}

END IF

// Add w to Visited list

Visited:= Visited union $\{\mathrm{w}\}$

// Update the minimum distance to vertices in remaining vertices

FOR each $\mathrm{u}$ in remaining vertices ShortestDistance $[\mathrm{u}]:=$

Minimum of

(ShortestDistance[u],ShortestDistance[w] + Distance $[\mathrm{w}, \mathrm{u}])$

\section{END FOR}

\section{END WHILE}

\subsection{Heuristic Algorithms (HA)}

When all the heuristic algorithms are concerned, A* algorithm can provide better results than the others when it comes to implementing them on real-time road network as $A^{*}$ algorithm uses a heuristic for searching best optimal route. The selection of node is done on the basis of calculation of cost from start node plus an estimate of cost to the destination. This project uses Euclidean distance as estimated distance to the destination.

The proposed strategy is a hybrid approach which takes full advantage of both Modified Dijkstra's Shortest Path (MDSP) algorithm and Heuristic algorithm (A* Search Method) and meets the requirements of dynamic time constraints of real road traffic scenarios. 


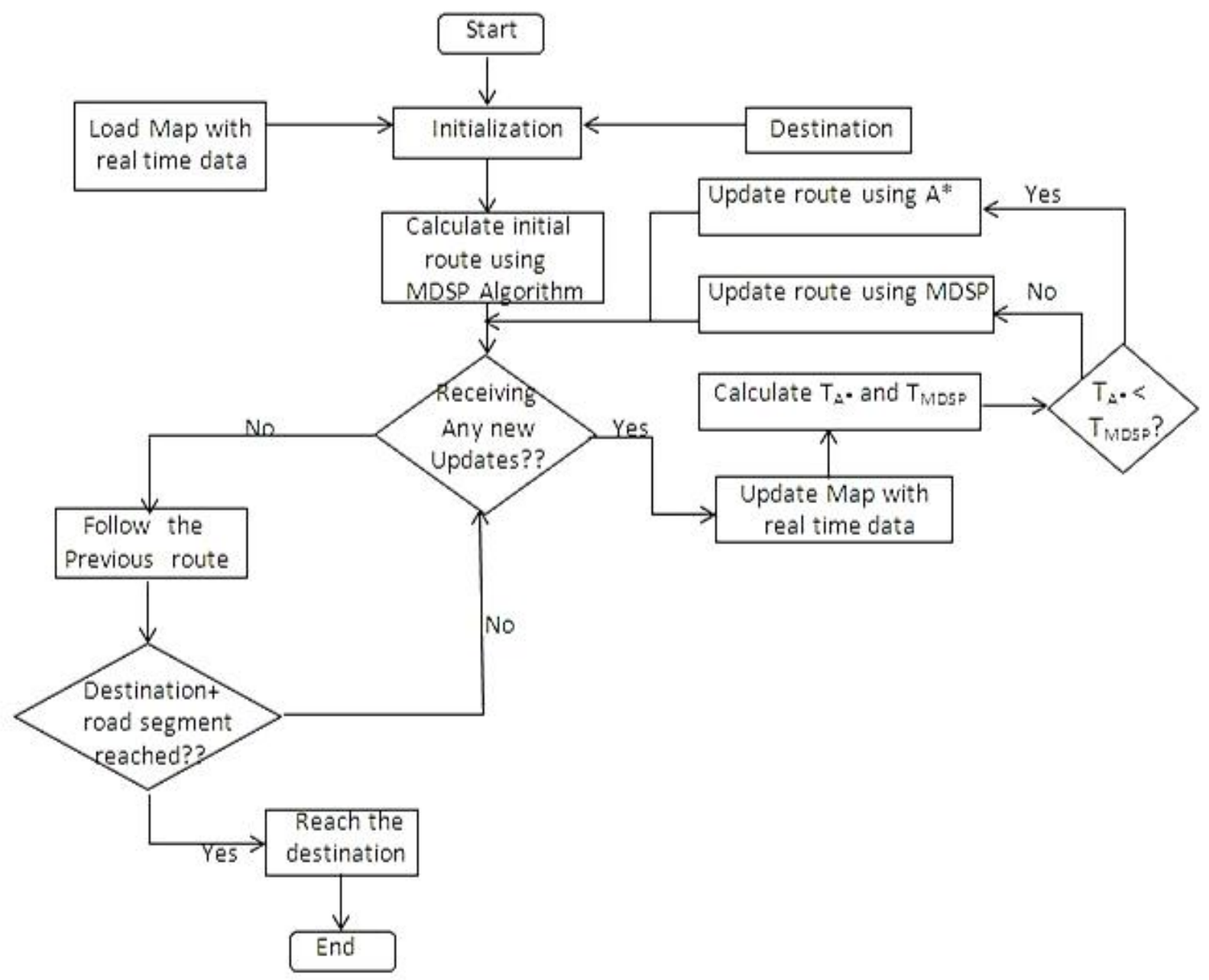

Fig 2: Proposed system flow diagram

- It's a strategy which uses a hybrid approach and takes full advantage of both MDSP and Heuristic algorithms (A* Search Method) and meets the requirements of dynamic time constraints of real road traffic scenarios.

- Initially, the shortest path is found using Modified Dijkstra's Algorithm. On receiving any new updates like new route, or change in congestion, or blockage of a road segment, an alternate route is calculated using A* Search Method and travel time of both the approaches are compared.

- The route with lower travel time period is chosen and route is updated accordingly.

- This process is repeated till the destination road segment is reached.

\section{SCOPE OF WORK}

The system is made up of four main modules and they are:

- Location Module

- Routing Module

- Speed Alerts

- Accident Alerts

\subsection{Location Module}

Google Map APIs are available for accessing the location from the device. These APIs are integrated within the software which allows one to access Google's Location Services. The device location is extracted in terms of latitude and longitude. The location module will trace the current location from the GPS enabled device without the need of user specifically entering it. The obtained latitude and longitude are converted into a specific location by GIS (Global Information System). After mapping this Location service, one can easily see the visual display of the road map with provided location.

\subsection{Routing Module}

Routing Module is the most important module of this project. It provides the driver with the path from the extracted location from location module to the destination which is supposed to be entered by the user. The main task of routing is not just to give the path, but to show the shortest possible distance from source to destination. For initial routing MDSP (Modified Dijkstra's shortest path) algorithm is used. The routing from a source to destination depends on various factors such as congestion, distance, time etc. If there is any disturbance in normal course of travel, there will be frequent update of routes. The route gets updated dynamically by applying the algorithm giving better results among MDSP and $\mathrm{A}^{*}$ algorithms. 


\subsection{Speed Alerts}

Providing speed alerts helps in maintaining security and safety of the kids as well as driver. This module has predefined threshold values depending on the road type. Whenever the driver exceeds the speed and crosses this threshold value, speed alert in terms of alarm notification is given in his cell phone. This notifies the driver that he needs to slow down the vehicle which has crossed the permissible speed limit.

The speed alert module comprises of various thresholds. These thresholds vary according to the various factors. Like highways have higher values as the roads are broader and congestion free; whereas the local streets have more congestion, so the threshold needs to be low. This improves the safety measure of the vehicles.

\subsection{Accident Alerts}

If the system detects a high probability of the occurrence of an accident, SMS is sent on urgent basis to the Vehicle Admin with the current location of the vehicle. Then, the vehicle admin is expected to find out the cause by contacting the driver and taking appropriate actions.

The occurrence of an accident can be noticed by the vehicle admin as the admin is watching over the complete journey of bus. The moment a vehicle stops moving for certain period, admin is expected to follow up about it as there is a chance of accident.

Also if accident occurs, driver is supposed to contact the admin regarding incident if he is able to. The admin will get the location of the place where the accident took place and then, he can take appropriate actions depending on the circumstances present.

\section{RESULTS ANALYSIS}

For this result analysis, an Android application was developed which took source and destination as inputs. By using different algorithms viz. Dijkstra's algorithm, MDSP, A* algorithm and the proposed algorithm, incorporated in the same application, shortest route statistics like distance and travel time were recorded using each of these algorithms as shown below:

Table 1. Record:1 (Charni Road-Ghatkopar)

\begin{tabular}{|c|c|c|}
\hline Algorithm & Distance & Travel Time \\
\hline Dijkstra's & $20 \mathrm{~km}$ & 51 minutes \\
\hline MDSP & $23 \mathrm{~km}$ & 44 minutes \\
\hline A* & $21 \mathrm{~km}$ & 49 minutes \\
\hline Proposed Algorithm & $23 \mathrm{~km}$ & 44 minutes \\
\hline
\end{tabular}

Table 2. Record:2 (Borivali - Kandivali)

\begin{tabular}{|c|c|c|}
\hline Algorithm & Distance & Travel Time \\
\hline Dijkstra's & $5.8 \mathrm{~km}$ & 26 minutes \\
\hline MDSP & $5.9 \mathrm{~km}$ & 24 minutes \\
\hline A* & $6.1 \mathrm{~km}$ & 25 minutes \\
\hline Proposed Algorithm & $5.9 \mathrm{~km}$ & 24 minutes \\
\hline
\end{tabular}

Table 3. Record:3 (Mumbai - Mahad)

\begin{tabular}{|c|c|c|}
\hline Algorithm & Distance & Travel Time \\
\hline Dijkstra's & $156 \mathrm{~km}$ & $\begin{array}{c}3 \text { hours } 42 \\
\text { minutes }\end{array}$ \\
\hline MDSP & $169 \mathrm{~km}$ & $\begin{array}{c}3 \text { hours } 27 \\
\text { minutes }\end{array}$ \\
\hline A* & $171 \mathrm{~km}$ & $\begin{array}{c}3 \text { hours } 24 \\
\text { minutes }\end{array}$ \\
\hline Proposed Algorithm & $171 \mathrm{~km}$ & $\begin{array}{c}3 \text { hours } 24 \\
\text { minutes }\end{array}$ \\
\hline
\end{tabular}

\section{CONCLUSION}

After successful implementation of the proposed project and deployment in the form of an android application, it will be much beneficial for the parents of school-going students. Right from the factors like safety of the students to the satisfaction of their parents, this system can be used by a school for giving assurance to the parents as it can monitor real-time position of the buses and even ensure that the driver does not over-speed. Also, the proposed system detects the occurrence of an accident with high probability so that immediate actions can be taken by the vehicle admin by looking into its details.

\section{REFERENCES}

[1] S. Sivakumar, Dr. C.Chandrasekar, "Modified Dijkstra's Shortest Path Algorithm" Ijir In Computer And Communication Engineering (An Iso 3297: 2007 Certified Organization)

[2] Liang Dai, "Fast Shortest Path Algorithm For Road Network And Implementation" Carleton University School Of Computer Science Honours Project Fall Term, 2005 Comp 4905

[3] A.Renugambal , V.Adilakshmi Kameswari ,"Finding Optimal Vehicular Route Based On Gps" ,Ijcsit, Vol. 5 (2) , 2014

[4] Paul Benjamin Et Al,"Design And Development Of Gps/Gsm Based Vehicle Tracking And Alert System For Commercial Inter-City Buses"

[5] M. A. Hannanet.Et Al,"Intelligent Bus Monitoring And Management System",Proceedings Of The World Congress On Engineering And Computer Science 2012

[6] Fleisher, Et Al,“Design And Development Of GPS/GSM Based Vehicle Tracking And Alert System For Commercial Inter-City Buses", Ijcsea. 